\title{
Hidden Markov Modeling of Human Normal Gait using Laser Range Finder for a Mobility Assistance Robot
}

\author{
Xanthi S. Papageorgiou, Georgia Chalvatzaki, Costas S. Tzafestas and Petros Maragos
}

\begin{abstract}
For an effective intelligent active mobility assistance robot, the walking pattern of a patient or an elderly person has to be analyzed precisely. A well-known fact is that the walking patterns are gaits, that is, cyclic patterns with several consecutive phases. These cyclic motions can be modeled using the consecutive gait phases. In this paper, we present a completely non-invasive framework for analyzing a normal human walking gait pattern. Our framework utilizes a laser range finder sensor to collect the data, a combination of filters to preprocess these data, and an appropriately synthesized Hidden Markov Model (HMM) for state estimation, and recognition of the gait data. We demonstrate the applicability of this setup using real data, collected from an ensemble of different persons. The results presented in this paper demonstrate that the proposed human data analysis scheme has the potential to provide the necessary methodological (modeling, inference, and learning) framework for a cognitive behavior-based robot control system. More specifically, the proposed framework has the potential to be used for the recognition of abnormal gait patterns and the subsequent classification of specific walking pathologies, which is needed for the development of a contextaware robot mobility assistant.
\end{abstract}

\section{INTRODUCTION}

\section{A. Motivation}

Mobility problems, particularly concerning the elderly population, constitute a major and ever growing issue in our society. Mobility disabilities are common and impede many activities of daily living important to independent living since they have strong impact in productive life, independence, physical exercise, and self-esteem, [1], [2]. Furthermore, current demographics show that the elderly population (aged over 65) in industrialized countries shows a constant increase, [3]. The social and economic significance of solving these issue should not be underestimated. Robotics seems to fit naturally to the role of assistance since it can incorporate features such as posture support and stability, walking assistance, navigation in indoor and outdoor environments, health monitoring etc.

The motivation in this work is to use intelligent mobile robotic mechanisms (e.g. a rollator, Fig. 1) which can monitor and understand specific forms of human walking activity in their workspace, in order to deduce their needs regarding mobility and ambulation, and to provide context-based sup-

This work is supported by $7^{\text {th }}$ Framework Program of the European Union, ICT Challenge 2, Cognitive Systems and Robotics, contract "EUFP7-ICT-2011-9 2.1 - 600796 - MOBOT: Intelligent Active MObility Assistance RoBOT Integrating Multimodal Sensory Processing, Proactive Autonomy and Adaptive Interaction".

The authors are with the School of Electrical and Computer Engineering, National Technical University of Athens, Greece, xpapag@mail.ntua.gr, gchal@central.ntua.gr, ktzaf, maragosecs.ntua.gr

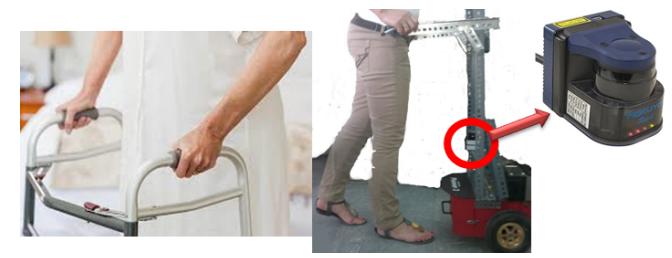

Fig. 1. Left: Typical passive assistive device for elderly. Right: A robotic platform based on Pioneer Robot equipped with a Hokuyo Laser Sensor aiming to record the gait cycle data of the user (below knee level).

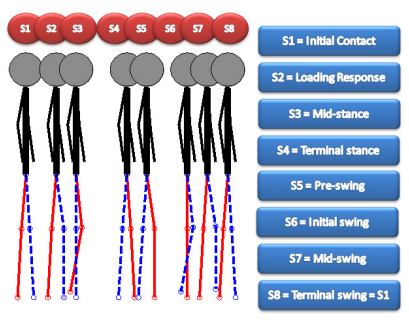

(a)

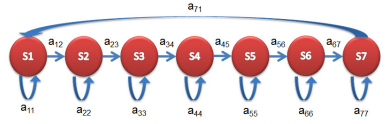

(b)
Fig. 2. (a) Internal states of normal gait cycle (Left Leg: blue dashed line, Right Leg: red solid line). (b) The topology of the network is a left-to-right Hidden Markov Model.

port, [4] and intuitive assistance in domestic environments.

In this paper we address the challenge of developing a reliable action recognition system by using non-wearable, completely noninvasive framework, utilizing a laser sensor (which does not interfere with human motion) and based on Hidden Markov Model (HMM) for gait representation and recognition. Our goal is to employ this setup as a subsystem within a larger behaviour-based robot control framework, which can be used for the development of a cognitive context-aware walking-aid robot.

\section{B. Related Work}

The automatic classification and modeling of specific physical activities of human beings is very useful for many technical and biomechanical applications. A number of research groups worldwide, are actively pursuing research, currently investigating problems related to the development of smart walking support devices, aiming to assist motorimpaired persons and elderly in standing, walking and other mobility activities, as well as to detect abnormalities and to assess rehabilitation procedures [5]-[11].

Different sensors have been employed for extracting gait motions such as foot pressure distributions (Smart Shoes), [12], joint angles and accelerations (gyroscopes, accelometers, [13], [14]), vision information (cameras, [15], 
[16]), myograohic activities (EMG-Electromyographic signals, [17]), etc. Most of these approaches refer's to human detection and tracking, or extraction of pedestrian patterns and recognition. There is also a growing body of research works utilizing laser finder sensors, and in some cases complementary with cameras, or force sensors, or other similar sensor, for problems of this kind [18]-[21].

Hidden Markov Models (HMMs) have been frequently used for modelling time series data in a wide range of applications, due to the fact that they are easy to build and manipulate, and there exist optimal algorithms to train them and score (e.g. maximum likelihood, Viterbi decoding). In a Markov model the states (corresponding to a physical event) of the model are visible to the observer. On the other hand, in HMMs, only the output of the model is visible and the states are not observable, in other words are hidden, [22]. The versatility of HMM makes them useful in extracting humans patterns. Apart from their prominent application in speech recognition, [23], HMMs are also used in pattern recognition applications such as biometric gait recognition, [24]-[28].

This paper proposes a flexible and readily extensible approach for gait representation and characterisation. In this work, an HMM is employed to represent normal human gait and to analyse transitions between specific gait phases. The human gait model used in this work is comprehensive, comprising a full eight-state representation. As opposed to most of the literature available on the topic, the gait analysis approach presented in this paper is completely noninvasive based on the use of a typical non-wearable device. Instead of using complex models and motion tracking approaches that require expensive or bulky sensors and recording devices that interrupt human motion, the observation data used in this work is provided by a standard laser rangefinder sensor mounted on a robotic rollator platform. This framework is designed so that it can be possible to actively incorporate many different normal gait patterns as a subsystem within a larger cognitive behaviour-based context-aware robot control framework (that embody several walking morphologies, including turning and maneuvering motions). Furthermore, this framework has the potential to be used for the recognition of abnormal gaits, for the classification of various walking pathologies and related impairments, and for actively and cognitively augmenting patients with mobility difficulties.

\section{NORMAL HUMAN GAIT CYCLE DESCRIPTION}

A basic requisite of the act of walking is the periodic movement of each foot from one position of support to the next. This element is necessary for any form of bipedal walking to occur, no matter how distorted the pattern may be by an underlying pathology, [29]. This periodic leg movement is the essence of the cyclic nature of human gait.

There are two main phases in the gait cycle, [30], [31]: The stance phase, when the foot is on the ground, and the swing phase when that same foot is no longer in contact with the ground and is swinging through in preparation for the next foot strike. The stance phase may be subdivided into three separate phases: 1. First double support, when both feet are in contact with the ground, 2. Single limb stance, when only one foot is in ground contact and the other foot is swinging forward, 3. Second double support, when both feet are again in ground contact. The same terminology would be applied for both the left and right side of the body. For a normal person, each side is half a cycle behind (or ahead of) the other side. Thus, first double support for the right side is second double support for the left side, and vice versa. In normal gait there is a natural symmetry between the left and right sides, but in pathological gait an asymmetrical pattern very often exists.

Traditionally the gait cycle has been divided into eight events or periods, five during stance phase and three during swing. The stance phase events are as follows, Fig. 2(a): 1. Heel strike initiates the gait cycle and represents the point at which the bodys centre of gravity is at its lowest position. 2. Foot-flat is the time when the plantar surface of the foot touches the ground. 3. Midstance occurs when the swinging (contralateral) foot passes the stance foot and the bodys centre of gravity is at its highest position. 4. Heel-off occurs as the heel loses contact with the ground and pushoff is initiated via the triceps surae muscles, which plantar flex the ankle. 5. Toe-off terminates the stance phase as the foot leaves the ground, [32]. The swing phase events are as follows: 6. Acceleration begins as soon as the foot leaves the ground and the subject activates the hip flexor muscles to accelerate the leg forward. 7. Midswing occurs when the foot passes directly beneath the body, coincidental with midstance for the other foot. 8. Deceleration describes the action of the muscles as they slow the leg and stabilize the foot in preparation for the next heel strike. Thus, there are eight events, but these are sufficiently general to be applied to any type of gait: 1 . Initial contact $(0 \%)-\mathbf{I C}, 2$. Loading response (0-10\%) - LR, 3. Midstance (10-30\%) - MS, 4. Terminal stance (30-50\%) - TS, 5. Preswing (50-60\%) - PW, 6. Initial Swing (60-70\%) - IW, 7. Midswing (70-85\%) MW, 8. Terminal swing (85-100\%) - TW, [30].

\section{DETECTION OF GAIT CYCLE BASED ON HIDDEN MARKOV MODEL}

\section{A. Hidden Markov Model for Human Normal Gait}

Hidden Markov Models are well suitable for gait recognition because of their statistical properties and its ability to reflect the temporal state-transition nature of gait. An HMM is defined as a doubly embedded stochastic process with an underlying process that is not observable (it is hidden), but can only be observed through another set of stochastic processes that produce the sequence of observations, [22], [23]. This reveals that the states underlying the data generation process are hidden, and they could be inferred through observations. HMMs are very common in several applications such as speech recognition, [23], [33], biological sequence analysis, [34], gesture recognition, [35], human activity analysis, [28], etc.

In this paper we have distinguished seven gait phases in order to analyze the normal gait cycle, since the Terminal Swing phase is characterized by heel strike that is equivalent 
trigger as for Initial Contact phase, and therefore TW and IC phases are treated as identical phases. These seven states can define the hidden states of the HMM. As observables, we utilize several quantities that represent the motion of the subjects' legs. These quantities (relative position w.r.t. the laser, velocities, etc.) are estimated using sequential signals from laser sensor installed on a Pioneer robot that follows the subject's motion. The state and observations at time $t$ are denoted as $s_{t}$ and $O_{t}$, respectively. The seven states at time $t=1,2, \ldots, T$, where $T$ is the total time, are expressed by the value of the (hidden) variable $s_{t}=i \in \mathbf{S}$, for $i=1, \ldots, 7$, where $1 \equiv I C / T W$ (since we treat IC and TW as identical), $2 \equiv L R, 3 \equiv M S, 4 \equiv T S, 5 \equiv P W, 6 \equiv I W$, and $7 \equiv M W$. Regarding observations at time $t$, we define nine signals denoted as $x^{m}, y^{m}, v_{x}^{m}, v_{y}^{m}$, for $m=\{R, L\}$, which are the coordinates and the velocities along the axis for right and left leg, respectively, and Dlegs which is the distance between legs, that are represented by the vector $O_{t}=\left[o_{t}^{1} \ldots o_{t}^{k}\right]^{T} \in \mathbf{O}$, for $k=1, \ldots, 9$, where $o_{t}^{1} \equiv x^{R}, o_{t}^{2} \equiv y^{R}, o_{t}^{3} \equiv x^{L}, o_{t}^{4} \equiv y^{L}$, $o_{t}^{5} \equiv v_{x}^{R}, o_{t}^{6} \equiv v_{y}^{R}, o_{t}^{7} \equiv v_{x}^{L}, o_{t}^{8} \equiv v_{y}^{L}$, and $o_{t}^{9} \equiv$ Dlegs.

Following the HMM notation, the transition probability matrix is defined as $A=\left\{a_{i j}\right\}$, where $a_{i j}=P\left[s_{t+1}=j \mid s_{t}=i\right]$, for $1 \leq i, j \leq N$, where $N$ is the number of states, i,e, the $(i, j)$ element of the matrix represents the transition probability from the $i^{\text {th }}$ state at a given time step to the $j^{\text {th }}$ state at the following time step. In the normal gait cycle the gait phases follow each other sequentially. Thus, this HMM is a leftto-right model. This means that the only feasible transitions from a state $i$ will be either to remain in the same state or to jump to the following adjacent state, as depicted in Fig. 2(b). The transition probability matrix, as well as the prior probability vector (i.e. the vector of probabilities $\pi_{i}$ of the system being at state $i$ at the initial time $t_{0}$ ), are estimated using the standard Baum-Welch algorithm, [23].

\section{B. Modeling the observation data}

The normal observation data (derived from the raw laser sensor data) are modeled using a mixture of Gaussian distributions This is a natural way of representing these data, as the data vector takes values from a bounded set (recall that we use the relative position of the legs from a robot that follows the subject with his/her mean velocity) and is inherently repetitive (due to the cyclic nature of the human gait). Thus, by collecting many data for a normal gait, we can obtain the mean and the variance of the Gaussian distributions of the mixture.

Since nine signals are measured and constitute the extracted features at each time instant, the distribution is a multivariate Gaussian distribution: $g\left(x \mid \mu_{m}, \Sigma_{m}\right)=$ $\frac{1}{(2 \pi)^{\frac{n}{2}}\left|\Sigma_{m}\right|^{\frac{1}{2}}} \exp \left\{-\frac{1}{2}\left(x-\mu_{m}\right)^{T} \Sigma_{m}^{-1}\left(x-\mu_{m}\right)\right\}$, where $x \in \mathbb{R}^{n}$ is the feature vector, $\mu_{m} \in \mathbb{R}^{n}$ is a mean vector, $\Sigma_{m}$ is a $(n \times n)$ covariance matrix, and in our case $n=9$, and $m=1, \ldots, M$ for a Gaussian Mixture Model (GMM). GMM is a weighted sum of $\mathrm{M}$ component Gaussian densities as given by the equation: $P(x)=\sum_{m=1}^{M} w_{m} \cdot g\left(x \mid \mu_{m}, \Sigma_{m}\right)$, where $w_{m}$ are the mixture weights, for which it holds: $\sum_{m=1}^{M} w_{m}=1, w_{m} \geq 0$.

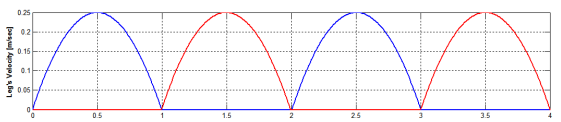

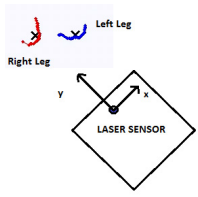

(a)
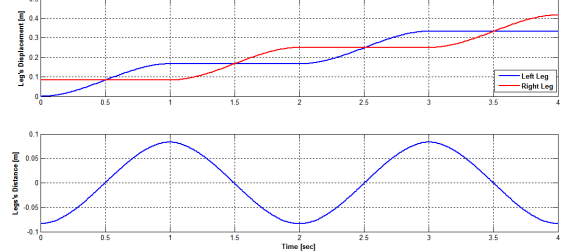

(b)
Fig. 3. (a) Left (blue data points) and Right (red data points) leg representation from laser data related to the Laser Sensor which is attached at the mobile robot. The black crosses indicate the centroid of the legs at each time instant. (b) Left (blue data points) and Right (red data points) leg representation for ideal gait cycle (top: velocities, middle: displacement, bottom: legs' distance in the sagittal plane).

\section{Estimation of state sequence}

The inference problem for an HMM is the following: given a sequence of observed data, compute the most probable sequence for the hidden states of the model, $p\left(s_{t} \mid \mathbf{O}\right)$, [23]. According to Bayes rule this probability for total experiment time $T$, can be estimated as: $p\left(s_{t} \mid \mathbf{O}\right)=$ $\frac{p\left(O_{1}, \ldots, O_{t} \mid s_{t}\right) \cdot p\left(O_{t+1}, \ldots O_{T} \mid s_{t}\right) \cdot p\left(s_{t}\right)}{p(\mathbf{O})}$. All these probabilities can be estimated recursively, based on transition probabilities, using the Viterbi algorithm [23].

\section{GAIT FEATURE VECTOR EXTRACTION}

\section{A. Feature variable selection}

The observation data used in the proposed HMM approach are defined by a set of gait feature variables, which are extracted based on the recordings provided continuously by a laser finder sensor. In order to use appropriate features to depict the gait motion, it is important to choose the simplest and most comprehensive data to describe the normal gait cycle. The ideal normal gait cycle in a straight line can be considered to follow the simple periodic motion pattern shown in Fig. 3(b) (adapted from [21]). From there it can be seen that the ideal normal gait follows some simple patterns. The first remark from this ideal gait cycle is the following: when the walking human starts moving the left leg, the right leg is stationary, and vice versa, which is reflected in the legs' velocities of the model. Therefore, the extracted velocity of the leg can convey information related to the gait phases. Another relevant remark that can be made from this observation is that when a leg holds its place (i.e. it is in a stance phase), it creates a stream of laser data points that are all approximately at the same position in a sequence of frames, which is reflected by the coordinates of each leg. Thus, the spatial coordinates of each leg, as depicted in the recording data, also provide important information that directly reflects the gait phases. Third, another important observation that can be made concerns distinguishing the "crossing points" between the legs, which are the points where the distance between the legs, at the sagittal plane, 
is zero (see Fig. 3(b)). This information is reflected on the displacement between the legs, and can also be used to characterize the gait phases. It is thus significant to estimate this information and use it in the gait features.

The above selected features (comprising velocities, positions, and interleg distance) are essential to characterize gait cycle properties and to signal transitions between gait phases. These features are extracted from the laser sensor data, as described in the following paragraph, and have been used as the observation data fed in the HMM.

\section{B. Feature vector estimation}

The above mentioned gait features are extracted using a laser range sensor, attached to a robot walker that follows the subject. The sensor is located at a height of $40 \mathrm{~cm}$ (approximately at the height of knee), scanning a plane parallel to the ground, as shown in Fig. 1. The robot walker follows the subject leaving an approximately constant clearance. The speed of the walker is the mean speed of the subject. The system records the relative position of the legs from the laser.

On these raw data, we employ a clustering technique (specifically a K-means clustering) to identify the legs of the subject, for each time instant, as indicated in Fig. 3(a). The centroid of the clusters is used as an estimate for the (relative) position of each leg. Using these data we compute the intraleg distance and, with a numerical smoothing and differentiation filter, we estimate the velocity of each leg. These values comprise the feature vector at each time instant that is used as observation data to the HMM.

\section{ANALYSIS AND EVALUATION OF EXPERIMENTAL RESULTS}

\section{A. Experiment and data description}

The data used in this paper were collected using a HOKUYO rapid laser sensor (UBG-04LX-F01, with mean sampling period of about $56 \mathrm{~ms}$ ) mounted on the Pioneer 3 mobile robot that was adapted with walking frame and handles to be used as the robotic walker during the experiment.

Ten subjects have participated in the experiment (three of them were aged under 30 years old, while the rest were aged over 30 years old, and six of the subjects were females). Each subject was capable to perform a normal walk, and was followed by the Pioneer robot in front of him/her. All the subjects were wearing tight clothes. There is no patient involvement in this study. In the experimental setup the robot was used as a robotic rollator. The length of the path was about $20 \mathrm{~m}$ down the hall on a flat marble surface. At the end of the corridor the subject had to turn around (the recordings of this turn were disregarded during preprocessing of the data), and then walk back the same distance. The subjects were instructed to walk as normally as possible. This results in a different walking speed for each subject, and in a different step length. The experimental data consists of only normal walks for each of the ten subjects.

Example of recorded data are depicted in Fig. 4. The upper part shows the velocity of both legs during the gait w.r.t. time. The second diagram corresponds to the evolution of

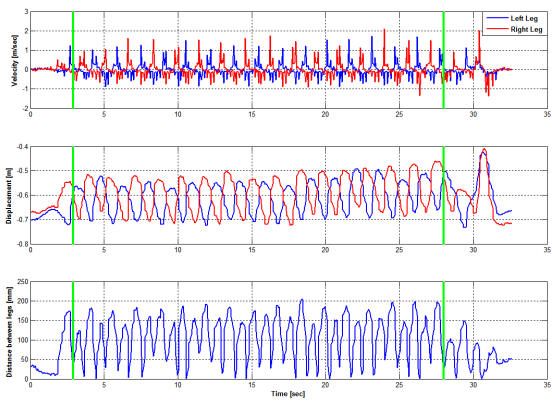

Fig. 4. Left (blue data) and Right (red data) leg representation from Real Experimental data (top: velocities, middle: displacement in the sagittal plane, bottom: legs's distance in the sagittal plane). The green vertical lines indicate the range of experimental data that are emitted to the HMM.

the displacement from the robotic rollator reference point in the sagittal plane, while in the bottom legs's distance in the sagittal plane is represented.

The experimental data that are used as observation data into HMM are divided into parts of $1.68 \mathrm{sec}$ (corresponds to a $9 \times 30$ observation data matrix). This time frame is empirically determined from the approximation of experimental time for each stride (which is the equivalent of gait cycle, i.e. two sequential steps define one stride, [30]) during the normal gait. Therefore, the total collected data during the experiment as divided above comprises 82 walking sections for all participated subjects (each walking section corresponds to one stride of $1.68 \mathrm{sec}$ ). Tests were done by using different number of Gaussian mixtures (as described in Subsection III-B). HMM training and testing was performed according to two different scenarios. According to Scenario $\boldsymbol{I}$ the HMM training phase includes all subjects' data (i.e. all the walking sections participate in the training). In Scenario 2, the training phase comprises only a part of subjects' data (only 76 of the walking sections are included in the training set), excluding the recorded data of one subject. This second scenario aims to test the performance of the proposed approach, validating its generalisation capacity over unseen data obtained by new subjects. Thus, two different models are trained, that is, one HMM for each scenario. The experimental results for both scenarios were done by using only one Gaussian mixture (for simplicity), and they are presented in the following subsection. The proposed algorithm was implemented in Matlab and it was running on an Intel(R) i5-2400 CPU @3.10GHz with 4GB RAM. The training phase took $13.5 \mathrm{sec}$, while the testing phase for new observation data took $35 \mathrm{msec}$.

\section{B. Experimental results}

The testing and evaluation phase of the HMMs is performed according to the two scenarios mentioned in the previous subsection. In both scenarios, evaluation is based on an assessment of the estimated states provided by the two constructed HMMs. Each HMM, after the training phase, represents human gait cycle. In the sequel, the model trained in the first case corresponding to a known scenario is called HMM1 (experimental test data is part of the training data 


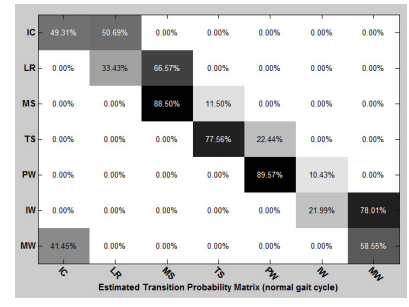

(a)

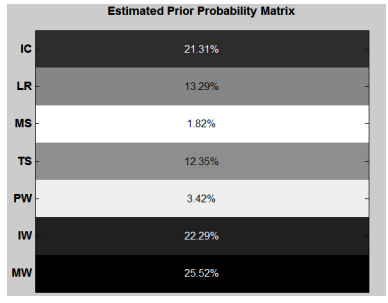

(b)
Fig. 5. (a) Estimated values of probability to jump from one state to the other. (b) Estimated values of prior probability that represent the probability of each initial state.

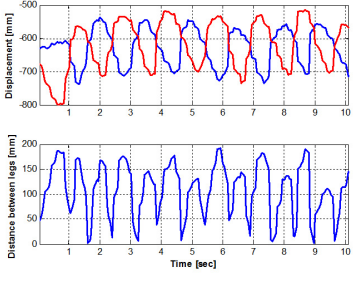

(a)

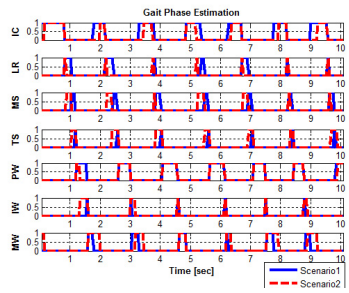

(b)
Fig. 6. (a) Real experimental data from one subject's normal gait that have been used in the testing and evaluation phase of both constructed HMMs. Top: Left (blue data) and Right (red data) legs displacement. Bottom: legs distance in the sagittal plane. (b) Scenario 1: Estimated sequence of gait states based on the constructed model (HMMI) with respect to time by testing the data depicted in Fig. 6(a) which represent a known walking section (blue line). Scenario 2: Estimated sequence of gait states based on the constructed model (HMM2) with respect to time by testing the data depicted in Fig. 6(a) which represent an unknown walking section (red line).

set), while the model trained in the second case referring to a completely unknown scenario is called $\boldsymbol{H M M 2}$ (the test set comprises new data not included in the training data set, and thus not used during the training phase of the model). The estimated values of the transition probability matrix $A$ are depicted in Fig. 5(a), while Fig. 5(b) shows the estimated values of prior probability vector.

The transition matrix $A$ in Fig. 5(a) has a triangular form. The diagonal elements represent the probabilities of selftransitions in gait phases, while the non-zero elements refer to the transition probabilities to the next adjacent gait phase only. The gait phases are changed with the correct sequence.

For testing and evaluation purposes of both constructed HMMs, the same real experimental data set has been used, which is depicted in Fig. 6(a). The goal of this evaluation phase is to unveil the hidden parts of the constructed models, i.e. to estimate the correct sequence of state transitions that occur in the test data. This test dataset reflects the normal gait of one subject and comprises 6 walking sections (about 6 strides). In this figure the displacement of each leg in the sagittal plane with respect to time is depicted on the top graph, while the bottom graph shows the evolution of the distance between legs within the same time frame. This figure is very useful to understand the exact subject's motion, as is described more in detail in the sequel.

The estimated sequence of gait states obtained using the model trained in the first scenario (i.e. using HMM1) is depicted in Fig. 6(b) with the blue line, while for the second scenario (i.e. by using $\mathrm{HMM} 2$ ) the respective results are presented again in Fig. 6(b) with the red line. A first remark that can be made by observing these experimental results is that the evolution of the gait states provided by both models matches the general evolution of the human normal gait model that is to be represented by the HMMs; i.e. the gait phases appear sequentially with the correct order, and the time frame of each state is within the general bounds as have been mentioned previously in Section II.

Another very significant result is that the HMM can take input data (observation data) without any restriction regarding the initial phase of the recordings and it can find the sequence of states from there on. This is very important in order to leave the subject free when starting to walk. There is an assumption, without a loss of generality, that at the beginning of each gait cycle the initial contact refers to the right leg, while a complete stride is concluded when the right leg is again in front of the left leg, ready for a new initial contact and therefore for the next stride. By observing the results depicted in Fig. 6(b), it can be seen that both models manage indeed successfully to recognize that (for the recorded experimental data of Fig. 6(a), used in this case study for model testing) the subject starts the motion with the right leg. Thus, the first estimated gait phase in Fig. 6(b) (for the first scenario) is Initial Contact (IC), while for the second scenario the first estimated phase is Midswing (MW).

Another remark concerns the terminal instant of the motion. At the end of the recorded test data of Fig. 6(a) (just after $10 \mathrm{sec}$ ) it can be seen that the motion is terminated approximately at the middle of a stride. Indeed, as shown in Figure 6(b), both models correctly recognise that the motion corresponding to the test data of Fig. 6(a) is terminated (just after 10sec) in a phase just after the middle of a gait cycle (PW in Fig. 6(b)). The results in both case studies show that constructed models recognize the normal gait, of course with different likelihood for each model (the sequence of phases has smaller probability in the second scenario when HMM2 is used). We assume as precision index the division of correctly detected states by all detected states, related to the reference data provided by scenario 1 . Therefore, the overlapping of those results between scenario 1 (which is the reference data) and scenario 2, in the form of precision index, is $90.95 \%$. Also, the time instances and duration of phases have variations, which is reasonable since in the case of the second scenario, the test data is completely unknown.

\section{CONCLUSIONS AND FUTURE WORK}

In this paper, a framework to represent and analyse human gait using a Hidden Markov Model (HMM) is presented. The HMM employed in this work analyses gait phases by using observation data provided by a typical laser rangefinder sensor, thus constituting a completely noninvasive approach using a non-wearable device. The proposed gait modeling method has been applied to actual human gait data. The experimental results clearly show that this method is capable of correctly recognizing human gait cycle patterns. Furthermore, this approach, based on its statistical learning 
properties, is quite flexible and readily extensible to different gait models, thus presenting a strong potential to support a behaviour-based cognitive robot control framework.

For further research, we will investigate the sequence of the gait phases in an abnormal gait. If the gait phases are not detected sequentially, then non-zero probabilities in the transition matrix can be observed between non-adjacent gait phases. Therefore, a variety of abnormal gaits (corresponding to specific motor impairments) can be characterized by the transition probabilities between different phases.

Furthermore, within our future plans is to model more gait patterns based on HMM, regarding turning motions during indoor ambulation, as well as more complicated and maneuvering motions that appear in daily activities. A data corpus will be created and annotated for this purpose, and the recorded data will be used to build different models for a variety of situations. The aim is to create a system that can detect in real time specific gait pathologies and automatically classify the patient status or the rehabilitation progress, thus providing the necessary information for effective cognitive (context-aware) active mobility assistance robots.

\section{REFERENCES}

[1] P. D. Foundation, "Statistics for parkinson's disease," 2010. [Online]. Available: http://www.pdf.org

[2] S. Center, "Stroke statistics," 2010. [Online]. Available: http://www.strokecenter.org

[3] USCensus, "The elderly population," 2010. [Online]. Available: http://www.census.gov

[4] P. Brezillon, "Context in problem solving: A survey," The Knowledge Engineering Review, vol. 14, pp. 1-34, 1999.

[5] H. Yu, M. Spenko, and S. Dubowsky, "An adaptive shared control system for an intelligent mobility aid for the elderly," Autonomous Robots, vol. 15, no. 1, pp. 53-66, 2003.

[6] S. Dubowsky, F. Genot, S. Godding, H. Kozono, A. Skwersky, H. Yu, and L. S. Yu, "Pamm - a robotic aid to the elderly for mobility assistance and monitoring: A 'helping-hand' for the elderly," in IEEE International Conference on Robotics and Automation, 2000, pp. 570576.

[7] M. Spenko, H. Yu, and S. Dubowsky, "Robotic personal aids for mobility and monitoring for the elderly," Neural Systems and Rehabilitation Engineering, IEEE Transactions on, vol. 14, no. 3, pp. 344-351, 2006.

[8] S. Jiang, B. Zhang, and D. Wei, "The elderly fall risk assessment and prediction based on gait analysis," in Computer and Information Technology (CIT), 2011 IEEE 11th International Conference on, 2011, pp. $176-180$.

[9] M. Bachlin, M. Plotnik, D. Roggen, I. Maidan, J. Hausdorff, N. Giladi, and G. Troster, "Wearable assistant for parkinson's disease patients with the freezing of gait symptom," Information Technology in Biomedicine, IEEE Transactions on, vol. 14, no. 2, pp. 436-446, 2010.

[10] A. Arcelus, I. Veledar, R. Goubran, F. Knoefel, H. Sveistrup, and M. Bilodeau, "Measurements of sit-to-stand timing and symmetry from bed pressure sensors," Instrumentation and Measurement, IEEE Transactions on, vol. 60, no. 5, pp. 1732-1740, 2011.

[11] J. F.-S. Lin and D. Kulic, "Automatic human motion segmentation and identification using feature guided hmm for physical rehabilitation exercises," in IEEE International Conference on Intelligent Robots and Systems, 2011.

[12] J. Bae and M. Tomizuka, "Gait phase analysis based on a hidden markov model," Mechatronics, vol. 21, no. 6, pp. 961 - 970, 2011.

[13] C. Nickel, C. Busch, S. Rangarajan, and M. Mobius, "Using hidden markov models for accelerometer-based biometric gait recognition," in Signal Processing and its Applications (CSPA), 2011 IEEE 7th International Colloquium on, 2011, pp. 58-63.

[14] I. Pappas, M. Popovic, T. Keller, V. Dietz, and M. Morari, "A reliable gait phase detection system," Neural Systems and Rehabilitation Engineering, IEEE Transactions on, vol. 9, no. 2, pp. 113-125, 2001.
[15] G. Bebis, M. Nicolescu, M. Nicolescu, A. Tavakkoli, C. King, and R. Kelley, "An architecture for understanding intent using a novel hidden markov formulation," International Journal of Humanoid Robotics, vol. 05, no. 02, pp. 203-224, 2008.

[16] A. Tavakkoli, R. Kelley, C. King, M. Nicolescu, M. Nicolescu, and G. Bebis, "A vision-based architecture for intent recognition," in Advances in Visual Computing, ser. Lecture Notes in Computer Science, G. Bebis, R. Boyle, B. Parvin, D. Koracin, N. Paragios, S.M. Tanveer, T. Ju, Z. Liu, S. Coquillart, C. Cruz-Neira, T. Muller, and T. Malzbender, Eds. Springer Berlin Heidelberg, 2007, vol. 4842, pp. $173-182$.

[17] M. Meng, Q. She, Y. Gao, and Z. Luo, "Emg signals based gait phases recognition using hidden markov models," in Information and Automation (ICIA), 2010 IEEE International Conference on, 2010, pp. 852-856.

[18] A. Panangadan, M. Mataric, and G. Sukhatme, "Tracking and modeling of human activity using laser rangefinders," International Journal of Social Robotics, vol. 2, no. 1, pp. 95-107, 2010. [Online]. Available: http://dx.doi.org/10.1007/s12369-009-0043-1

[19] H. Kim, W. Chung, and Y. Yoo, "Detection and tracking of human legs for a mobile service robot," in Advanced Intelligent Mechatronics (AIM), 2010 IEEE/ASME International Conference on, 2010, pp. 812817.

[20] W.-H. Mou, M.-F. Chang, C.-K. Liao, Y.-H. Hsu, S.-H. Tseng, and L.C. $\mathrm{Fu}$, "Context-aware assisted interactive robotic walker for parkinson's disease patients," in Intelligent Robots and Systems (IROS), 2012 IEEE/RSJ International Conference on, 2012, pp. 329-334.

[21] X. Shao, H. Zhao, K. Nakamura, R. Shibasaki, R. Zhang, and Z. Liu, "Analyzing pedestrians' walking patterns using single-row laser range scanners," in Systems, Man and Cybernetics, 2006. SMC '06. IEEE International Conference on, vol. 2, 2006, pp. 1202-1207.

[22] L. Rabiner and B. Juang, "An introduction to hidden markov models," ASSP Magazine, IEEE, vol. 3, no. 1, pp. 4 -16, Jan. 1986.

[23] L. R. Rabiner, "Readings in speech recognition," A. Waibel and K.-F. Lee, Eds. San Francisco, CA, USA: Morgan Kaufmann Publishers Inc., 1990, ch. A tutorial on hidden Markov models and selected applications in speech recognition, pp. 267-296. [Online]. Available: http://dl.acm.org/citation.cfm?id=108235.108253

[24] B.-C. Cheng, Y.-A. Tsai, G.-T. Liao, and E.-S. Byeon, "Hmm machine learning and inference for activities ofdaily living recognition," The Journal of Supercomputing, vol. 54, no. 1, pp. 29-42, 2010.

[25] C. Chen, J. Liang, H. Zhao, and H. Hu, "Gait recognition using hidden markov model," in Advances in Natural Computation, ser. Lecture Notes in Computer Science, L. Jiao, L. Wang, X.-b. Gao, J. Liu, and F. Wu, Eds. Springer Berlin Heidelberg, 2006, vol. 4221, pp. 399407.

[26] A. Kale, A. N. Rajagopalan, N. Cuntoor, and V. Kruger, "Gait-based recognition of humans using continuous hmms," in Automatic Face and Gesture Recognition, 2002. Proceedings. Fifth IEEE International Conference on, 2002, pp. 336-341.

[27] A. Mannini and A. M. Sabatini, "Accelerometry-based classification of human activities using markov modeling," p. 10, 2011.

[28] P. Turaga, R. Chellappa, V. S. Subrahmanian, and O. Udrea, "Machine recognition of human activities: A survey," Circuits and Systems for Video Technology, IEEE Transactions on, vol. 18, no. 11, pp. 14731488,2008

[29] R.-H. T. F. Inman, V.T., Human walking. Baltimore: Williams \& Wilkins, 1981.

[30] J. Perry, Gait Analysis: Normal and Pathological Function. Slack Incorporated, 1992.

[31] J. C. O. Christopher L. Vaughan, Brian L. Davis, Dynamics of Human Gait. Human Kinetics Publishers, 1992.

[32] G. Cochran, A primer of orthopaedic biomechanics. New York: Churchhill Livingstone, 1982.

[33] A. Katsamanis, G. Papandreou, and P. Maragos, "Audiovisual-toarticulatory speech inversion using active appearance models for the face and hidden markov models for the dynamics." in ICASSP. IEEE, 2008, pp. 2237-2240.

[34] B.-J. Yoon, "Hidden markov models and their applications in biological sequence analysis." Curr Genomics, vol. 10, no. 6, pp. 402-15, 2009.

[35] S. Theodorakis, A. Katsamanis, and P. Maragos, "Product-hmms for automatic sign language recognition.” in ICASSP. IEEE, 2009, pp. 1601-1604. 